\title{
Neural correlates of inter-trial priming and role-reversal in visual search
}

\author{
Christopher Rorden ${ }^{1,2}{ }^{*}$, Arni Kristjansson ${ }^{3}$, Kathleen Pirog Revill ${ }^{1}$ and Styrmir Saevarsson ${ }^{1,4}$ \\ ${ }^{1}$ Center for Advanced Brain Imaging, Georgia Institute of Technology, Atlanta, GA, USA \\ ${ }^{2}$ Department of Psychology, McCausland Center for Brain Imaging, University of South Carolina, Columbia, SC, USA \\ ${ }^{3}$ Department of Psychology, University of Iceland, Reykjavik, Iceland \\ ${ }^{4}$ Neuropsychology Research Group (EKN), Municipal Clinic Munich GmbH, Bogenhausen University Hospital, Munich, Germany
}

\section{Edited by:}

Kenneth Hugdahl, University of

Bergen, Norway

Reviewed by:

Stefan Pollmann, Otto von Guericke University, Germany

John J. McDonald, Simon Fraser

University, Canada

${ }^{*}$ Correspondence:

Christopher Rorden, McCausland

Center for Brain Imaging, University

of South Carolina, Columbia, SC

29208, USA.

e-mail: rorden@sc.edu
Studies of priming of visual perception demonstrate that observers respond more quickly to targets in a field of distractors when relevant features are repeated versus novel or rolereversed. In a recent brain imaging study by Kristjánsson et al. (2007), participants were presented with two items of one color and a single item in a different color with the task of reporting the orientation of the uniquely colored item. Consistent with previous behavioral reports, they found that observers were faster to respond when the target and distractor colors were identical to the previous trial than when they were reversed. They found reduced BOLD activity in brain areas linked with attentional control on trials where the target and distractor colors were repeated relative to reversed, which they interpreted as reflecting response suppression (decreased BOLD signal for repeated stimuli). However, since their design only compared repeated versus reversed task demands, it is logically possible that this pattern reflects increased BOLD signal for role-reversed stimuli: activity required to inhibit previously facilitated information and select previously inhibited information. We explored this possibility with a task where we contrasted the signal generated by repeated, reversed, and novel features. Our data suggest that the majority of the change in neural signal elicited by priming of pop-out reflects increased activation when selection criteria are reversed.

Keywords: psychophysics, priming of pop-out, human vision, attention, repetition suppression

\section{INTRODUCTION}

Visual attention effectively identifies relevant targets from displays cluttered with irrelevant distractors. The processes behind this have been subject to intense research. An influential study by Maljkovic and Nakayama (1994) described the "priming of popout" (PoP) effect which reveals inter-trial priming during pop-out visual search, and how attention and vision can be modulated by events in the past. Observers are faster and more accurate (Kristjánsson and Driver, 2008) at identifying a feature of a discrepant target (e.g., the orientation of a red item where multiple green items are present) if the target feature is repeated (target was red on previous trial) relative to reversed (target was green on previous trial). These effects are found even though the current target color holds no predictive value about upcoming target and distractor colors (see however Tanaka and Shimojo, 1996 and Campana and Casco, 2009 for null results on spatial position repetition). Note that in the paradigm the behavioral response (e.g., the discrepant item's orientation) is independent of the feature that defines the item as a target (its color). While inter-trial priming effects had been previously demonstrated (see Hillstrom, 2000 for review), Maljkovic and Nakayama's paradigm has provided a popular tool for a wide range of behavioral research. The effect has been heavily replicated and, along with other perceptual phenomena such as probability cueing, inhibition of return, and contextual cueing, is beginning to provide a deeper understanding of the facilitatory and inhibitory processes that guide perceptual selection (see Kristjánsson, 2008 for review). The neurological basis of this effect has recently been investigated using brain imaging (Kristjánsson et al., 2007; see also Geng et al., 2006), electrophysiology (Eimer et al., 2010), neuropsychology (Kristjánsson et al., 2005; Saevarsson et al., 2008), and brain stimulation (Campana et al., 2006; Muggleton et al., 2010). Our aim was to replicate a previous brain imaging study by Kristjánsson et al. (2007) but most importantly extend the inferences from that study by including additional control conditions.

Examining the neurological correlates of PoP can provide new insight into how the human perceptual system operates. For example, electrophysiological recordings by Eimer et al. (2010) demonstrate that inter-trial priming influences the early $\mathrm{N} 2 \mathrm{pc}$ components, indicating at least a portion of the PoP effect reflects early perceptual/attentional stages of processing. Recent work by Saevarsson et al. (2008) demonstrates that individuals who experience visual neglect following brain injury exhibit relatively spared PoP effects for both targets and distractors, providing additional evidence for the theory that PoP is influenced by low-level perceptual changes (see also Kristjánsson et al., 2005).

Kristjánsson et al. (2007) examined the brain regions involved with the PoP effect in a functional magnetic resonance imaging study. This technique is sensitive to blood oxygenation levels, and is used to infer brain activation with relatively high spatial resolution 
(relative to other non-invasive measures). On each trial they presented a display with three diamonds, one directly above fixation and one in the left and right lower quadrants. The top diamond was either red or green, and one of the lower items was the same color as the top item (a distractor), whereas the other was a color singleton (green if the other diamonds were red and red if the other diamonds were green). Each of the lower items had a notch removed from either the top or bottom, and the participants' task was to simply report the position of the singleton's notch. Unpredictably, in half of the trials the colors of the target and distractors were the same as in the previous trial, whereas in the other half the colors were reversed (likewise, the location of the target was either repeated or reversed). The authors reported classical behavioral PoP effects: observers were slower to respond on trials where target and distractor colors reversed than when they were repeated. Crucially, they observed less brain activity bilaterally on trials where the color mapping was repeated relative to trials where the mapping reversed (their Table 3). Specifically, this modulation was observed in three regions classically identified in brain imaging studies of endogenous visual attention (see de Haan et al., 2008 for review): the frontal eye fields (FEF), the intraparietal sulcus (IPS), and the lateral occipital cortex (as well as activity in the left frontal gyrus and right occipital cortex).

Kristjánsson et al. (2007) interpreted their findings as demonstrating that repetition of target and distractor color leads to reduced activity in the attentional network. This explanation fits nicely with well-documented repetition suppression of BOLD signal as task features are repeated compared to when they are novel (Buckner et al., 1998). It is common in the literature to interpret inter-trial changes in BOLD signal as reflecting response suppression rather than response enhancement (for review, see Grill-Spector et al., 2006). But there is another potential explanation. A similar pattern of brain activity would have been found if target-distractor reversal yields increased activation. Indeed, these explanations are not mutually exclusive, and therefore some brain regions may show modulated activity due to feature repetition, others due to feature reversal, and some areas may exhibit both effects. Specifically, similar to Maljkovic and Nakayama's (1994) seminal behavioral work, Kristjánsson et al. (2007) only contrast repetition versus switching, making it impossible to ascertain if the effect reflects primarily response suppression due to repetition facilitation, primarily response enhancement due to switching costs, or a mixture. As recently noted in behavioral studies (Geyer et al., 2006; Kristjánsson and Driver, 2008; Lamy et al., 2008), these effects can be teased apart by introducing trials where both targets and distractors can be repeated, reversed, or novel (e.g., using a paradigm where stimuli can be presented in three or more colors, such that the target and/or the distractor can be defined by a color not used in the previous trial). A drawback to this approach is that it generates a much larger set of conditions, reducing statistical power. This is especially serious for conventional voxelwise fMRI studies, where thousands of independent statistical tests are conducted which require some form of correction for multiple comparisons (e.g., familywise error control or false discovery rate). To address this, we focused on the regions of interest identified by Kristjánsson et al. (2007), allowing us to make strong a priori predictions regarding the brain regions involved with PoP. We also defined regions of interest based on the orthogonal contrast of trial (regardless of condition) relative to rest, and then examined whether these regions differentiate across conditions. Such independent region of interest analyses pool data across relevant anatomical regions, and dramatically reduce the number of statistics computed. Both these approaches improve statistical power relative to voxelwise analysis (Poldrack, 2007). Our aim was to identify which of these regions showed feature repetition-related suppression effects, and which exhibit role-reversal enhancement.

\section{MATERIALS AND METHODS BEHAVIORAL TASK}

Our behavioral task was an extension of Kristjánsson et al. (2007), with the added inclusion of novel distractor and target colors. Specifically, in their study the stimuli were either red or green (presented on a black background). In contrast, we used four equiprobable colors displayed on a gray background (8-bit red, green, blue $=128,128,128)$ : white $(255,255,255)$, black $(0,0,0)$, blue $(0,0,255)$, and yellow $(255,255,0)$. These colors were chosen in pilot validation of the task in order to generate maximum distinctiveness between stimuli. While many studies of PoP have used stimuli with matched contrast or luminance, previous studies have shown robust effects with different contrasts (Kristjánsson et al., 2008; Ásgeirsson and Kristjánsson, 2011). We developed customized software that used the computer's video card (NVidia GeForce 9800) and the OpenGL library to ensure accurate frame rates.

On each trial, three diamond-shaped stimuli were presented radially distributed around a central white fixation dot, one directly above fixation, one in the lower right field and one in the lower left field. Each diamond had a notch removed from either the top or bottom (independent of each other). One of the lower items always matched the color of the top item, whereas one always had a unique color. These diamonds were only visible for $200 \mathrm{~ms}$ (precisely 12 frames for our 60-hz displays) to discourage eye movements, and participants were instructed to maintain fixation at the center throughout the study. On each trial, participants reported whether the notch was located at the top or bottom of the oddly colored diamond by pressing a button with the middle finger or index finger (corresponding to notch at top or bottom button, respectively), regardless of position or color.

Trials were blocked into 201 trial sessions (first trial discarded from analysis). The trials were pseudo-randomized so that there were precisely 40 instances of each of our five conditions which were defined based on the color of items relative to the previous trial. We describe these conditions following the convention of Lamy et al. (2008), using the nomenclature repeated (" $r$ "), novel/new ("n"), and switched ("s") for both the target (T) and distractors (D): rTnD (target repeated), sTnD (target was previously distractor), $\mathrm{nTsD}$ (distractor was previously a target), $\mathrm{nTrD}$ (distractor repeated), and $\mathrm{nTnD}$ (distractors and target novel). Example trials are shown in Figure 1.

The mean inter-trial interval was $3 \mathrm{~s}$ on average, pseudorandomly selected with equal probability from the continuous range $2-4 \mathrm{~s}$. This jittered presentation rate reduces participant temporal expectancy and provides a better estimate of hemodynamic response relative to rest. 


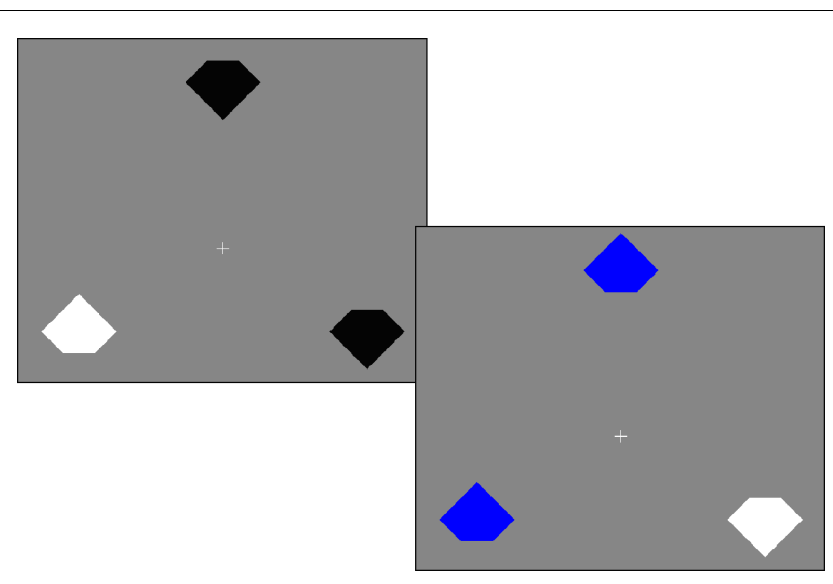

FIGURE 1 | Example trials. On each trial, the participant's task is to report the orientation of the notch for the item with the odd color. In the first trial the target is white and the distractors are black, whereas in the second trial the target feature is repeated (the target is still white), but the distractor feature is novel (blue). Therefore, we refer to the second trial as $r T n D$ (repeated target, novel distractor). Priming of pop-out refers to effects where people are faster for repeated features and slower when roles are reversed (e.g., the feature that designated a distractor in the previous trial now indicates a target).

All observers performed one practice session in a mock scanner. The experiment parameters were identical to the actual scan, with stimuli visible on a liquid crystal display (a Hewlett-Packard L1506 that mimics the resolution and aspect ratio of the MRIcompatible projector) that was viewable using a mirror attached to the mock head coil.

Each individual performed four sessions of the task while in the MRI scanner: one during the initial anatomical scan followed by three sessions during fMRI acquisition. Stimuli were presented using an Avotec Silent Vision 6011 projector using its native resolution $(1024 \times 768)$. Each notched diamond subtended $1.8^{\circ}$ horizontally and $1.4^{\circ}$ vertically. The center of each diamond was positioned $4^{\circ}$ from the fixation. An optical pulse from the scanner initiated data collection in the scanner, with the first trial included in the statistical analysis occurring approximately $9 \mathrm{~s}$ into the acquisition, minimizing any T1-saturation effects in our fMRI data.

\section{PARTICIPANTS}

Twenty-four healthy volunteers (eight females) with normal or corrected-to-normal visual acuity participated in this study, following protocols and a consent procedure approved by the Joint Georgia State and Georgia Tech Center for Advanced Brain Imaging Institutional Review Board. The mean age was 22 years (SD: 3.73). One participant performed poorly on the behavioral task (51\% accuracy, not significantly different from chance as determined by binomial probability), and two exhibited excessive head movement during scanning ( $>5 \mathrm{~mm}$ translation; one performed at $75 \%$ accuracy and the other at $89 \%$ ). All data from these three individuals was excluded from the subsequent analyses. All remaining participants performed at $94 \%$ accuracy or higher and had head translations of $4 \mathrm{~mm}$ or less.

\section{MAGNETIC RESONANCE IMAGING ACQUISITION AND OTHER EQUIPMENT}

All MRI data were collected on a Siemens $3 \mathrm{~T}$ Trio scanner with a 12-channel RF-receive head coil, with images manually aligned to the AC-PC horizontal plane. Localizer and T1-weighted images were acquired during the first $10 \mathrm{~min}$ in the scanner. The T1 scan was used to normalize the shape of each individual's brain to standard stereotaxic space. Specifically, we used a 3D MP-RAGE sequence with a TI of $900 \mathrm{~ms}$, a TR of $2250 \mathrm{~ms}$ between TFE shots and a $9^{\circ}$ flip angle, TE of $4.52 \mathrm{~ms}, 1601 \mathrm{~mm}$ sagittal slices each with a $256 \times 256$ matrix, and $256 \mathrm{~mm} \times 256 \mathrm{~mm}$ FOV. Next, the participant completed three sessions of the task during continuous fMRI acquisition. Each session lasted $610 \mathrm{~s}$, allowing us to acquire 305 3D fMRI volumes per session. This T2*-weighted echo planar imaging pulse sequence included the following parameters: repetition time, $2.0 \mathrm{~s}$; echo time, $32 \mathrm{~ms}$; flip angle, $90^{\circ}$; $68 \times 68$ matrix; $204 \mathrm{~mm} \times 204 \mathrm{~mm}$ field of view; 36 ascending $3.2 \mathrm{~mm}$ thick slices with $10 \%$ slice gap, resulting in voxels with an effective distance of $3 \mathrm{~mm} \times 3 \mathrm{~mm} \times 3.52 \mathrm{~mm}$ between voxel centers.

\section{DATA ANALYSIS}

Data from the practice session in the mock scanner were excluded from the analysis. As mean reaction times are influenced by outliers, we excluded trials that were faster or slower than 2 SDs from the mean in each condition. Repeated-measures statistics were computed using ezANOVA ${ }^{1}$.

Imaging data was analyzed with $\mathrm{SPM}^{2}$ and the free automated analysis scripts from Cambridge Neuroimaging ${ }^{3}$. Raw DICOM format images were converted to NIfTI using SPM8. Data preprocessing included motion correction and spatial smoothing using a 8-mm FWHM Gaussian kernel, as well as a high pass $($ sigma $=128 \mathrm{~s})$ temporal filter. The fMRI images were coregistered to each individual's structural T1-weighted image, which was normalized to the T1 template image included with SPM using both linear and non-linear deformation. The resulting parameters were used to reslice each individual's scan to standard space with a resolution of $3 \mathrm{~mm}$ isotropic. Individual statistical analysis included local autocorrelation correction, with session number, temporal derivatives, and motion parameters added as covariates of no interest. Because the location of functional modules varies slightly between individuals, we generated spherical regions of interest with a $16-\mathrm{mm}$ diameter, centered on the coordinates reported by Kristjánsson et al., 2007, their Table 3). For each individual we computed the mean $t$-score for $10 \%$ of voxels within the region. The location of a module might differ across individuals, so the region of interest serves as a mask for the $t$-score maps with only the most significant voxels in each region contributing. Note that we select the top $10 \%$ individually for each condition versus rest (e.g., sTnD > rest, etc.), so there is not a consistent bias for the subsequent analyses between conditions \{consider the contrast Repeat $<$ Novel in Table 2: this is based on each individual's mean $t$-score for the two repeat conditions $(\mathrm{rTnD}>$ rest) and $(\mathrm{n} \operatorname{TrD}>$ rest $)$ versus the $t$-score for the $(\mathrm{nTnD}>$ rest condition $)$,

\footnotetext{
${ }^{1}$ http://www.mricro.com/ezanova/

${ }^{2}$ http://www.fil.ion.ucl.ac.uk/spm/

${ }^{3}$ http://www.cambridgeneuroimaging.com/
} 
such that each condition represents $20 \%$ of the trials, whereas the comparison $[(\mathrm{rTnD}$ or $\mathrm{nTrD})>$ rest] would be expected to have higher $t$-scores as it is influenced by $40 \%$ of the trials\}. These measures were extracted from the regions of interest values using MRIcron software (Rorden et al., 2007).

\section{RESULTS}

\section{BEHAVIORAL TASK}

A one-factor analysis of variance (ANOVA) for reaction time revealed a main effect of condition $F(4,80)=3.38, p<0.0013$. We subsequently conducted planned comparisons of the behavioral data without correcting for multiple comparisons as we had clear a priori predictions with respect to effects and directions of effects, wanted to maximize statistical power and our main thrust was to examine neural changes rather than behavioral changes (as in theory even subtle behavioral differences may reflect robust neural differences in processing). Numerically, the trends were as predicted by the PoP effect, with faster responses as color is repeated. Specifically, repeated targets $(\mathrm{rTnD})$ were responded to faster $(718 \mathrm{~ms})$ than switched targets $(728 \mathrm{~ms}$; sTnD), and trials where the distractor was repeated (702 ms; nTrD) were responded to faster than trials where the distractor was switched (711 ms; nTsD). The mean response time for trials where both the target and distractors were novel (nTnD) was $718 \mathrm{~ms}$. Post hoc repeated-measures $t$-tests were used to evaluate the main effect of condition (see Figure 2). Reaction times were faster for trials in which distractors were repeated $(702 \mathrm{~ms} ; \mathrm{nTrD})$ than for trials in which both the target and distractor were novel [718 ms; nTnD; $t(20)=2.93, p<0.0083]$, as well as for trials where the target was previously the distractor [728 ms; $\mathrm{sTnD} ; t(20)=4.27, p<0.0004]$.

For the error rates, there was no main effect of condition $F(4,80)=0.701, p=0.594$, and none of the post hoc $t$-tests were significant at $p<0.05$. However, the numerical trend for error rates was as predicted by the PoP effect: repeated information $(\mathrm{rTnD}$, $3.33 \% ; \mathrm{nTrD}, 2.86 \%$ ) tended to be responded to more accurately than switched information (sTnD, 3.45\%; nTsD, 3.02\%). Curiously, completely novel displays (nTnD) had the highest error rate $(3.53 \%)$, potentially reflecting a general benefit for familiar information relative to novel information.

A more direct way to examine the behavioral influence of feature repetition versus feature switching is a two-way ANOVA, with the factors of feature (two levels: was the target or distractor carried over from the previous trial) and change (two levels: was the feature repeated or switched). This two-by-two analysis excludes the $\mathrm{nTnD}$ condition. This analysis reveals a significant effect of feature $[F(1,20)=6.75, p<0.017, \mathrm{MSE}=834.40]$ and a strong trend for change $[F(1,20)=4.31, p<0.051, \mathrm{MSE}=464.64$, note this is a two-tailed $p$-value, whereas we had a strong prediction regarding the direction], but an interaction between these factors was not confirmed $[F(1,20)=0.019, p<0.89, \mathrm{MSE}=794.83]$. Specifically, the response times suggest participants were faster when distractors were carried over (mean $706 \mathrm{~ms}$ ) than when the target was

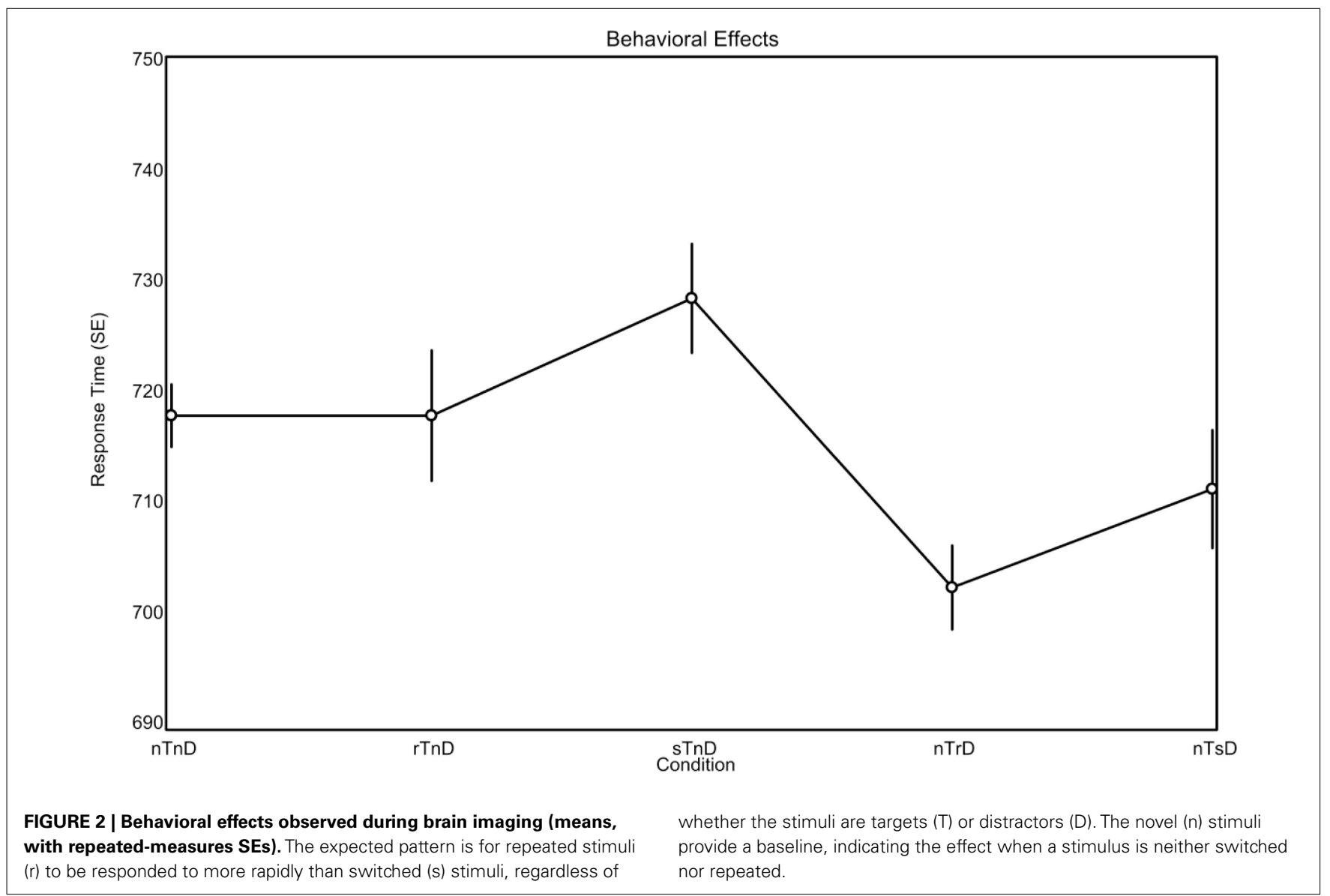


carried over (723 ms). Crucially, the observers responded faster when an item role was repeated $(710 \mathrm{~ms})$ than when an item role was reversed (720 ms), which is the classic PoP effect, and numerically, the performance for when both the target and distractor was novel fell between these two values $(718 \mathrm{~ms})$. A similar analysis of error rates identified no significant effects.

In order to increase statistical power and provide more direct comparison with the work of Kristjánsson et al. (2007) we collapsed the conditions into three effects: repeat trials $(\mathrm{r} T \mathrm{nD}, \mathrm{n} \operatorname{TrD})$, novel trials (nTnD), and switch trials (sTnD, nTsD), with the strong a priori behavioral prediction that the best performance would be seen for repeat trials, followed by novel trials, with the worst performance for switch trials. Numerically, the RTs fit this pattern $(710,718,720 \mathrm{~ms}$ for repeat, novel, switch). We conducted $t$-tests between conditions ( $\mathrm{df}=20$, where $t=1.725$ is the one tailed $p<0.05)$ and found that observers responded faster on repeat trials than on novel trials $[t(20)=1.97$ ], while performance on novel trials was not significantly different from switch trials $[t(20)=0.43]$. Finally, we found that observers responded faster on repeat trials than switch trials $[t(20)=2.08]$. No differences were found for error rates (all $t<1$ ).

\section{IMAGING DATA}

The functional imaging data was analyzed to identify regions that exhibit trial-related activity (regardless of trial condition), using a threshold of $p<0.05$ corrected for familywise error $(\mathrm{df}=20$, threshold $=6.586)$. This voxelwise analysis (Table 1; Figure 3) revealed bilateral activations in the sensory motor, superior frontal, intraparietal, anterior insula, and occipital cortices as well as medial activation in the supplementary motor and anterior cingulate regions.

To identify the neural correlates for PoP, we used a priori regions of interest based on the previous study ROIs, using the top $10 \%$ of $t$-scores as described earlier (Table 2). Figure 4 shows the overlap between these ROIs and our voxelwise analysis. Our attempt to replicate their contrasts is the switch $>$ repeat effect $[(\operatorname{sinD}+n T s D)>(r \operatorname{TnD}+n \operatorname{TrD})]$, where we found significant effects in the predicted direction in the left IPS, right IPS, and left lateral occipital area. Note importantly that our addition of a novel condition (nTnD) allows us to determine whether this effect reflects activation increases from role-reversals or decreased activity from repetition. None of the regions showed the pattern of decreased activation to repeated information inferred by Kristjánsson et al. (2007) (i.e., no region showed statistically significant lower $t$-scores for the repeated versus novel condition). On the other hand, the left IPS, left FEF, and right FEF all showed significantly increased activation for switch trials relative to novel trials. This effect is illustrated in Figure 5, which for completeness shows the $t$-scores for all five conditions.

We conducted an additional region of interest analysis based on the voxelwise analysis of our own study. Note that our voxelwise analysis identifies regions that are active during any condition relative to rest, whereas our subsequent region of interest analysis of these regions looks at whether activity observed in these regions differentiates between conditions. This region of interest analysis (Table 3) identified the left IPS and right IPS as demonstrating enhanced activity in switch trials relative to novel trials.

\section{DISCUSSION}

Our analysis of the behavioral data reveals a subtle but reliable influence of inter-trial feature repetition, replicating the extensive behavioral literature investigating PoP (see, e.g., Kristjánsson and Campana, 2010, for review; though note we failed to detect behavioral target facilitation, in contrast to studies such as Lamy et al., 2008). Our primary aim was to explore the neural correlates for this effect, using a design that allows us to disentangle repetition benefits of feature repetition versus switch costs from role-reversal. Kristjánsson et al. (2007) only compared these two conditions, while our inclusion of a novel condition allows us to detect whether changes in activation measures reflect decreased

Table 1 | Regions more active during trials (regardless of condition) than rest at $p<0.05$ corrected for familywise error (threshold $t>6.58$ ).

\begin{tabular}{llllll}
\hline Region & Cluster size & Peak $\boldsymbol{t}$ & \multicolumn{2}{l}{ Coordinates } \\
\hline Left sensorimotor cortex, & 1331 & 14.31 & -45 & -28 & 46
\end{tabular}

superior frontal, intraparietal,

and medial supplementary

motor, and anterior cingulate

Left premotor

Left anterior insula

Right anterior insula

Right intraparietal sulcus

Right inferior frontal gyrus

Right lateral occipital gyrus

Left lateral occipital gyrus

$\begin{array}{rrrrr}119 & 13.68 & -51 & 8 & 31 \\ 52 & 10.79 & -30 & 26 & 1 \\ 82 & 10.01 & 33 & 23 & 1 \\ 74 & 9.22 & 33 & -43 & 49 \\ 14 & 8.62 & 54 & 8 & 22 \\ 22 & 8.29 & 36 & -76 & 31 \\ 10 & 7.55 & -27 & -76 & 28\end{array}$

All reported clusters have a corrected $p<0.001$. Cluster size, peak $t$-score (degrees of freedom $=20$ ), and coordinates in MNI space are also provided.

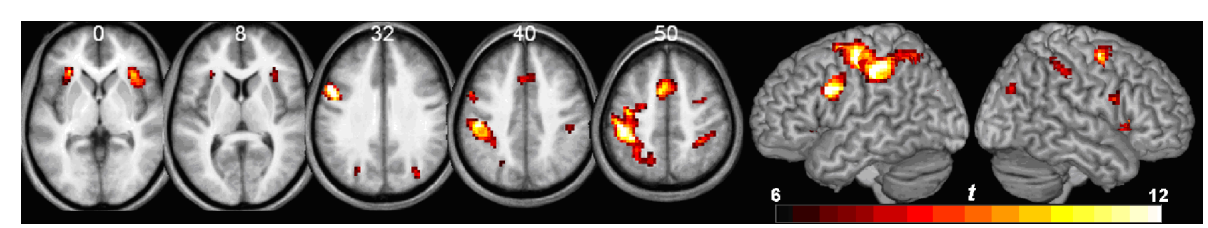

FIGURE 3 | Voxelwise analysis showing regions that show trial-related activity (regardless of condition), $\boldsymbol{p}<\mathbf{0 . 0 5}$ corrected for multiple comparisons. Results are shown in neurological orientation (with left presented on the left). Numbers above axial slices denote $Z$ dimension of
MNI space. Note bilateral activity in the anterior insula, supplementary motor area, and intraparietal cortex, with a largely left-lateralized somatosensory response (probably reflecting the fact that participants made right-handed responses). 
Table 2 | Region of interest analysis.

\begin{tabular}{|c|c|c|c|c|c|c|}
\hline Region & repeat & novel & switch & repeat $<$ novel & switch > novel & switch $>$ repeat \\
\hline Left intraparietal sulcus & 3.71 & 3.68 & 3.81 & -0.73 & $2.31^{*}$ & $1.95^{*}$ \\
\hline Right intraparietal sulcus & 3.12 & 3.15 & 3.22 & 0.60 & 1.00 & $2.11 *$ \\
\hline Right FEF & 3.09 & 3.08 & 3.18 & -0.25 & $1.97^{*}$ & 1.59 \\
\hline Left lateral occipital & 2.16 & 2.20 & 2.23 & 1.01 & 0.48 & $2.15^{*}$ \\
\hline Right lateral occipital & 0.74 & 0.71 & 0.72 & -0.75 & 0.49 & -0.29 \\
\hline Left frontal gyrus & 1.71 & 1.67 & 1.74 & -0.78 & 1.05 & 0.73 \\
\hline
\end{tabular}

The locations of our a priori regions of interest were derived from Kristjánsson et al. (2007). Each row shows measures for a different region. The first column provides the name for the region, and the next three columns report the $t$-score for that region (the mean value for the $10 \%$ most active voxels in that region) for each of the

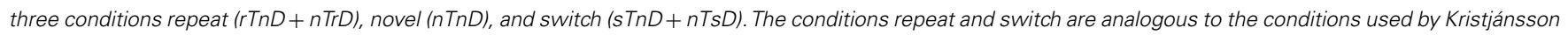

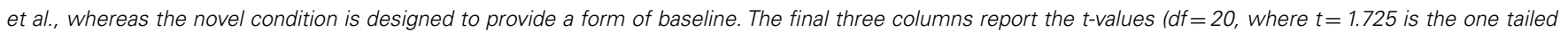

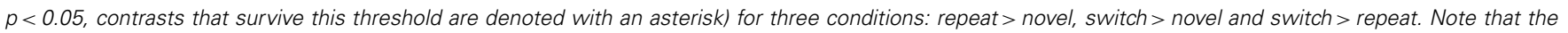

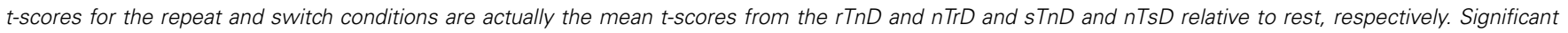

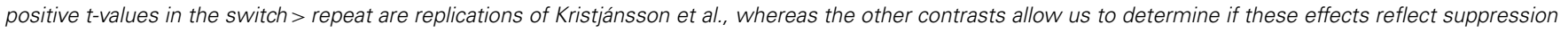
or enhancement. FEF refers to "frontal eye fields" - the superior frontal sulcus).

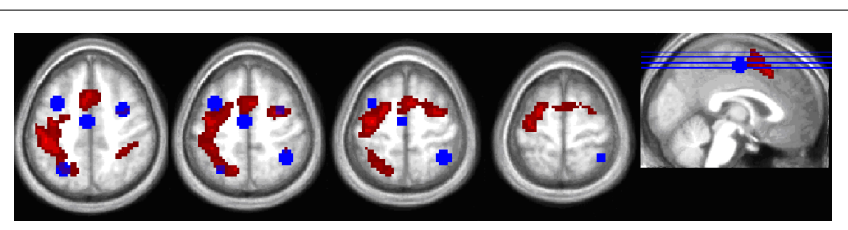

FIGURE 4 | Overlap between the thresholded trial-related activity from the voxelwise analysis (hot colors) and the ROIs defined from the peak activation coordinates in Kristjánsson et al. (2007) for their color repeat $<$ switch contrast (in blue).

activity from search task repetition or increased signal from search task switching. Our analyses suggests that the primary effects in terms of blood oxygenation related signal reflect increased activation in response to role-reversal (role-reversal leads to response enhancement). While "response suppression" has proved to be a useful interpretation for many neuroimaging findings, our work emphasizes the need to ensure that the effects do not actually reflect "response enhancement" (Henson and Rugg, 2003).

When we restrict our analysis to analogous conditions as in Kristjánsson et al. (2007) we appear to replicate their findings. Specifically, consider the "switch $>$ repeat" contrast in Tables 2 and 3. Thirteen of the 14 contrasts show numerical trends in the predicted direction, and five of these reached our statistical threshold, specifically, the left IPS, right IPS, and left lateral occipital regions reported in the previous study and the left IPS and the right IPS regions defined by our orthogonal voxelwise analysis (Figure 1). However, as noted, this contrast does not distinguish between BOLD response suppression and response enhancement. Therefore, while these contrasts tend to replicate the previous findings, analysis of other conditions allowed us to test their inference of these effects.

In theory, brain regions could show both response suppression due to repetition facilitation (reduced BOLD response for repeated versus novel features) as well as response enhancement due to the cost of inhibition (increased activation for switched versus novel features). However, inspection of Tables 2 and 3 provides no evidence for response suppression: none of the 14 regions of interest show significance for the predicted "repeat < novel" contrast, and 7 of the 14 show numerical trends in the opposite direction (i.e., these regions tended to show more activation for repeated features than novel features). On the other hand, five regions showed significant response enhancement (increased activity) in the "switch $>$ novel" contrast, and all 14 regions showed numerical trends in this direction. Specifically, regions that showed more activation for switch relative to novel were the left IPS, left FEF, and right FEF regions defined by the previous study of Kristjánsson et al. (2007) and the left and right IPS regions defined by our orthogonal voxelwise contrast. Taken together, this provides compelling evidence that feature changes induce increased neural responses relative to novel features. Activity increases in FEF, lateral occipital cortex, and IPS are typically seen when observers make voluntary eye movements or when they are asked to attend to peripheral information while suppressing eye movements (Corbetta et al., 2008).

We identified several regions that were activated by our task but where the pattern of activation did not distinguish between different inter-trial patterns, consistent with the notion that these regions do not play a major role in the PoP effect (though null effects are hard to interpret). For example, we propose that the activation in the left primary somatosensory cortex reflects the right-handed response required in our task. In addition, we identified activation in the bilateral anterior insula and a medial region that includes the anterior cingulate and neighboring portions of the medial prefrontal cortex. These regions appear to work as a network, as many neuroimaging tasks identify these areas together. For example, these regions are consistently activated during different tasks that all require a level of response inhibition (Wager et al., 2005). In a recent review, Craig (2009) describes a wide 


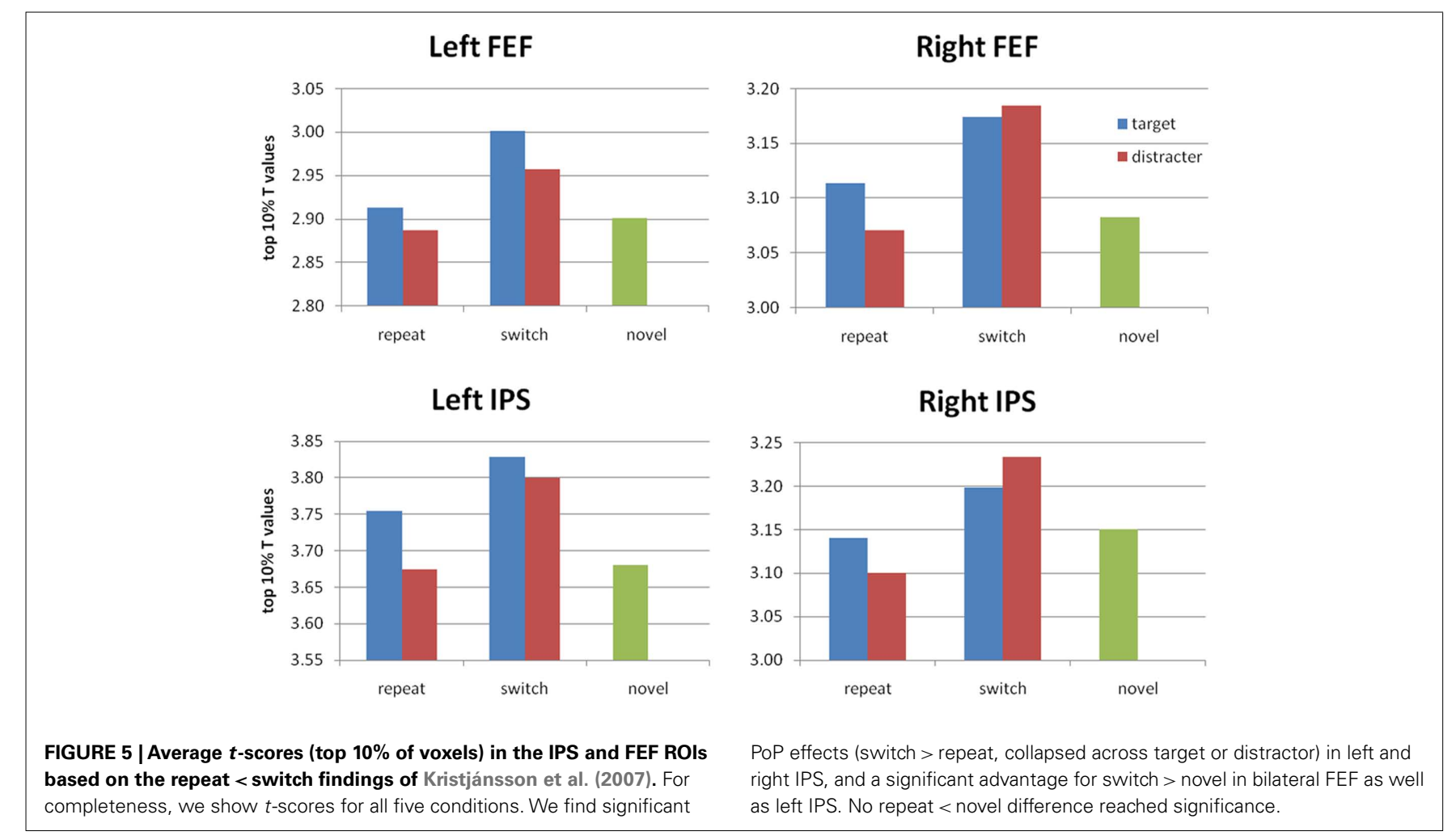

Table 3 | Region of interest analysis.

\begin{tabular}{lllllll}
\hline Region & repeat & novel & switch & repeat $<$ novel & switch $>$ novel & switch $>$ repeat \\
\hline Left intraparietal sulcus & 3.89 & 3.85 & 3.99 & -0.97 & $2.53^{*}$ & $2.32^{*}$ \\
Right intraparietal sulcus & 3.83 & 3.86 & 3.97 & 0.65 & $1.79^{*}$ & $2.67^{*}$ \\
Lateral occipital gyrus (bilateral) & 3.07 & 3.04 & 3.12 & -0.98 & 1.62 & 0.89 \\
Anterior insula (bilateral) & 3.58 & 3.60 & 3.61 & 0.36 & 0.10 & 0.47 \\
Premotor (bilateral) & 4.45 & 4.47 & 4.50 & 0.59 & 0.46 & 0.78
\end{tabular}

These regions are functionally defined as regions more active during trials (regardless of condition) than rest at a corrected $p<0.05$ (see Table 1). Left IPS was defined

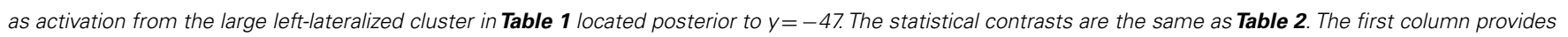

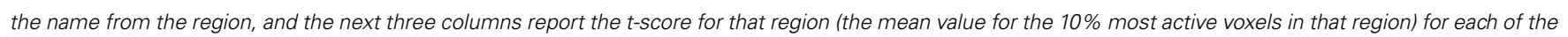

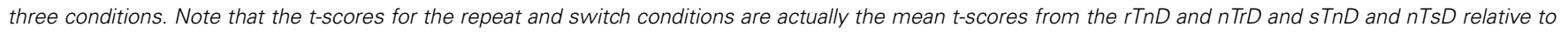
rest, respectively. The final three columns report the $t$-values $(d f=20$, where $t=1.724$ is $p<0.05$ ).

range of paradigms that elicit activation of this circuit, and speculates that this region is activated by tasks that requiring some level of self-awareness. Therefore, these regions do not appear to be specifically selective for particular tasks, making the interpretation of activity in this circuit challenging. However, we speculate this may reflect response inhibition in our tasks (where some trials require inhibition of a prepotent response).

Here we focused on the neural consequences of modulating target color (novel, repeated, or switched). However, we note that the preceding work examined the effects of color (repeated versus switched) and position (repeated versus switched). The paradigm we use here (including novel features) could be extended to spatial position, to further reveal if the effects observed previously reflect enhancement or suppression. While the earlier study emphasized the similarity of the neural effects observed, it is logically possible that the there may be different patterns of facilitation and inhibition for features versus locations. There is indeed behavioral evidence that these mechanisms may be dissociable, and that the patterns of inhibition and facilitation may be influenced by the type of response required (e.g., detection, orientation discrimination, position discrimination; Tanaka and Shimojo, 1996; Campana and Casco, 2009). Further, this discussion necessarily focuses on the statistically significant findings of our neuroimaging study. Therefore, we are unable to speculate on several interesting potential effects such as the difference between novel and repeat conditions. These null results may reflect the relatively low statistical power inherent to neuroimaging, the small effect size of our behavioral paradigm (as our behavioral effects are numerically smaller than several previous studies), differential neural responses with similar amplitude across conditions, or 
the fact that there is truly little neural difference between these conditions. Clearly, future studies could tailor the frequency of events and manipulate the behavioral paradigm to address these issues.

We believe that our findings make strong prediction for future brain stimulation studies. Whereas brain activation measures identify regions involved with a task, brain disruption methods can identify which regions of the brain are crucial for generating a normal PoP effect. Indeed, careful reading of the brain stimulation literature does seem to suggest that the FEF may be more involved with inhibition for switched trials rather than facilitation of repeated information. For example, Figure 4 of Campana et al. (2007) suggests that stimulation of the left FEF leads to faster responses on trials where the position is switched without influencing response time on trials where position is repeated (Campana et al., 2006 show similar effects for area V5/MT for priming of motion detection). Therefore, the general pattern from brain stimulation studies suggests that disruption can facilitate

\section{REFERENCES}

Ásgeirsson, Á. G., and Kristjánsson, Á. (2011). Episodic retrieval and feature facilitation in intertrial priming of visual search. Atten. Percept. Psychophys. 73, 1350-1360.

Buckner, R. L., Goodman, J., Burock, M., Rotte, M., Koutstaal, W., Schacter, D., Rosen, B., and Dale, A. M. (1998). Functional-anatomic correlates of object priming in humans revealed by rapid presentation event-related fMRI. Neuron 20, 285-296.

Campana, G., and Casco, C. (2009). Repetition effects of features and spatial position: evidence for dissociable mechanisms. Spat. Vis. 22, 325-338.

Campana, G., Cowey, A., Casco, C., Oudsen, I., and Walsh, V. (2007). Left frontal eye field remembers "where" but not "what." Neuropsychologia 45, 2340-2345.

Campana, G., Cowey, A., and Walsh, V. (2002). Priming of motion direction and area V5/MT: a test of perceptual memory. Cereb. Cortex 12, 663-669.

Campana, G., Cowey, A., and Walsh, V. (2006). Visual area V5/MT remembers "what" but not "where." Cereb. Cortex 16, 1766-1770.

Corbetta, M., Patel, G., and Shulman, G. L. (2008). The reorienting system of the human brain: from environment to theory of mind. Neuron 58 , 306-324.

Craig, A. D. (2009). How do you feel now? The anterior insula and human awareness. Nat. Rev. Neurosci. 10, 59-70.

de Haan, B., Morgan, P. S., and Rorden, C. (2008). Covert orienting of attention and overt eye movements activate identical brain regions. Brain Res. 1204, 102-111.
Eimer, M., Kiss, M., and Cheung, T. (2010). Priming of pop-out modulates attentional target selection in visual search: behavioural and electrophysiological evidence. Vision Res. 50, 1353-1361.

Geng, J. J., Eger, E., Ruff, C. C., Kristjánsson, A., Rotshtein, P., and Driver, J. (2006). On-line attentional selection from competing stimuli in opposite visual fields: effects on human visual cortex and control processes. J. Neurophysiol. 96, 2601-2612.

Geyer, T., Muller, H., and Krummenacher, J. (2006). Cross-trial priming in visual search for singleton conjunction targets: role of repeated target and distractor features. Percept. Psychophys. 68, 736-749.

Grill-Spector, K., Henson, R., and Martin, A. (2006). Repetition and the brain: neural models of stimulusspecific effects. Trends Cogn. Sci. (Regul. Ed.) 10, 14-23.

Henson, R. N., and Rugg, M. D. (2003). Neural response suppression, haemodynamic repetition effects, and behavioural priming. Neuropsychologia 41, 263-270.

Hillstrom, A. P. (2000). Repetition effects in visual search. Percept. Psychophys. 62, 800-817.

Kristjánsson, Á. (2008). "I know what you did on the last trial" - a selective review of research on priming in visual search. Front. Biosci. 13, 1171-1181.

Kristjánsson, Á., and Campana, G. (2010). Where perception meets memory: a review of priming in visual search. Atten. Percept. Psychophys. 72, 5-18.

Kristjánsson, Á., and Driver, J. (2008). Priming in visual search: separating

responses in switch trials (Campana et al., 2002, 2006, 2007; O'Shea et al., 2007; though see Muggleton et al., 2010). This work not only demonstrates that these brain regions are crucial for the PoP effect, but also supports our suggestions that some brain areas play a dominant role in inhibition of information.

Our current work suggests that future stimulation studies guided by our coordinates and using tasks that include novel trials should be able to shed further light on these components. In particular, we suggest that the IPS plays an important role in remapping features during the reversal trials, such that stimulating this region should drastically influence performance on switch trials. Furthermore, it would be interesting to disentangle switch and repetition for other features such as color and location in stimulation and brain imaging studies.

\section{ACKNOWLEDGMENTS}

This work was supported by the National Institutes of Health (R01 NS054266).

the effects of target repetition, distractor repetition and role-reversal. Vision Res. 48, 1217-1232.

Kristjánsson, Á., Ingvarsdóttir, Á., and Teitsdóttir, U. D. (2008). Objectand feature-based priming in visual search. Psychon. Bull. Rev. 15, 378-384.

Kristjánsson, Á., Vuilleumier, P., Malhotra, P., Husain, M., and Driver, J. (2005). Priming of color and position during visual search in unilateral spatial neglect. J. Cogn. Neurosci. 17, 859-873.

Kristjánsson, Á., Vuilleumier, P., Schwartz, S., Macaluso, E., and Driver, J. (2007). Neural basis for priming of pop-out during visual search revealed with fMRI. Cereb. Cortex 17, 1612-1624.

Lamy, D., Antebi, C., Aviani, N., and Carmel, T. (2008). Priming of popout provides reliable measures of target activation and distractor inhibition in selective attention. Vision Res. 48, 30-41.

Maljkovic, V., and Nakayama, K. (1994). Priming of pop-out: I. Role of features. Mem. Cognit. 22, 657-672.

Muggleton, N. G., Juan, C. H., Cowey, A., Walsh, V., and O'Breathnach, U. (2010). Human frontal eye fields and target switching. Cortex 46, 178-184.

O'Shea, J., Muggleton, N. G., Cowey, A., and Walsh, V. (2007). Human frontal eye fields and spatial priming of pop-out. J. Cogn. Neurosci. 19, 1140-1151.

Poldrack, R. A. (2007). Region of interest analysis for fMRI. Soc. Cogn. Affect. Neurosci. 2, 67-70.

Rorden, C., Bonilha, L., and Nichols, T. E. (2007). Rank-order versus mean based statistics for neuroimaging Neuroimage 35, 1531-1537.

Saevarsson, S., Jóelsdóttir, S., Hjaltason, H., and Kristjánsson, A. (2008). Repetition of distractor sets improves visual search performance in hemispatial neglect. Neuropsychologia 46, 1161-1169.

Tanaka, Y., and Shimojo, S. (1996). Location vs feature: reaction time reveals dissociation between two visual functions. Vision Res. 36, 2125-2140.

Wager, T. D., Sylvester, C. Y., Lacey, S. C., Nee, D. E., Franklin, M., and Jonides, J. (2005). Common and unique components of response inhibition revealed by fMRI. Neuroimage 27, 323-340.

Conflict of Interest Statement: The authors declare that the research was conducted in the absence of any commercial or financial relationships that could be construed as a potential conflict of interest.

Received: 27 September 2011; accepted: 09 November 2011; published online: 29 November 2011.

Citation: Rorden C, Kristjansson A, Revill KP and Saevarsson S (2011) Neural correlates of inter-trial priming and role-reversal in visual search. Front. Hum. Neurosci. 5:151. doi: 10.3389/fnhum.2011.00151

Copyright (c) 2011 Rorden, Kristjansson, Revill and Saevarsson. This is an openaccess article distributed under the terms of the Creative Commons Attribution Non Commercial License, which permits non-commercial use, distribution, and reproduction in otherforums, provided the original authors and source are credited. 